\title{
CAPACIDADE FUNCIONAL, ETIOLOGIA E LOCAL ANATÔMICO DA LESÃO NO ACIDENTE VASCULAR CEREBRAL
}

\author{
FUNCTIONAL CAPACITY, ETIOLOGY AND ANATOMIC INJURY IN LOCAL STROKE
}

\author{
Natália Rosa e Souza ${ }^{\mathrm{a}^{*}}$, Luis Paulo Nunes Caldeira ${ }^{\mathrm{b}^{* *}}$, Omar Pereira de Almeida Neto ${ }^{\mathrm{c}^{* *}}$, \\ Maria Elizabeth Roza Pereira ${ }^{\mathrm{d}^{* *}}$ \\ anatalia.r.souza@hotmail.com, bluispaulo_caldeira@hotmail.com, comarpneto@hotmail.com, delizebthp@famed.ufu.br \\ *Universidade Federal do Triângulo Mineiro - Uberaba (MG), Brasil \\ **Universidade Federal de Uberlândia - Uberlândia (MG), Brasil
}

\section{RESUMO}

Introduçáo: Apesar dos avanços tecnológicos e científicos cerebrovasculares, o acidente vascular cerebral (AVC) ainda é sinônimo de morbimortalidade e incapacidade funcional. Objetivos: Descrever o perfil clínico e socioeconômico e a capacidade funcional de pacientes pósAVC, assim como o tipo e o local anatômicos do acometimento. Método: Estudo transversal, quantitativo e descritivo, realizado no ambulatório de neurologia da Universidade Federal de Uberlândia. Após o consentimento dos participantes, foram realizadas entrevistas clínicas e sociodemográficas e aplicação da Escala de AVC dos National Institutes of Health e do Índice de Barthel Modificado. Este estudo foi aprovado pelo comitê de ética da instituição, sob no 277.825. Resultados: Foram recrutados 14 pacientes. O tipo de AVC mais prevalente foi o hemorrágico $(64,28 \%)$, acometendo o lobo frontal $(42,85 \%)$, seguido do lobo parietal $(28,59 \%)$. A maioria dos indivíduos era independente para se locomover $(64,28 \%)$, porém com dificuldades para realização de atividades de vida diária (78,57\%). A Escala de AVC dos National Institutes of Health evidenciou acometimento moderado (57,14\%). Conclusáo: A relação entre o AVC do tipo hemorrágico e a capacidade funcional relaciona-se com déficits neurológicos mais graves, em regiôes como lobos cerebrais frontal e parietal.

Palavras-chave: Acidente vascular cerebral; manifestaçôes neurológicas; estatísticas de sequelas e incapacidade.

\section{ABSTRACT}

Background: Despite technological and cerebrovascular scientific advances, stroke is still synonymous with mortality and functional disability. Objectives: To describe the clinical and socioeconomic profile and the functional capacity of post-stroke patients, as well as the type and anatomical site of involvement. Methods: Crosssectional, quantitative and descriptive study, held in AVC Clinic of the Federal University of Uberlândia. After the consent of the participants were conducted clinical and sociodemographic interviews and application of the National Institutes of Health Stroke Scale and the Barthel Index Modified. This study was approved by the ethics committee of the institution under no. 277,825. Results: We recruited 14 patients. The most prevalent type of stroke was hemorrhagic (64.28\%), affecting the frontal lobe $(42.85 \%)$, followed by the parietal lobe $(28.59 \%)$. Most individuals were independent to get around (64.28\%), but having difficulty performing activities of daily living (78.57\%). The National Institutes of Health Stroke Scale showed moderate involvement (57.14\%). Conclusion: The relationship between stroke hemorrhagic type and the functional capacity relates to more severe neurological deficits in regions such as the frontal and parietal brain lobes.

Keywords: Stroke; neurologic manifestations; sequels and disability statistics. 


\section{Introdução}

$\mathrm{O}$ acidente vascular cerebral (AVC) é definido como uma síndrome com desenvolvimento rápido de sinais clínicos e perturbação focal ou global da função cerebral, de possível origem vascular, com mais de 24 horas de duração e com aspecto repetitivo do déficit neurológico, o que causa uma série de comprometimentos motores e sensoriais no paciente acometido ${ }^{1}$.

Dados divulgados pela Organização Mundial de Saúde (OMS) revelam a incidência de seis milhóes de mortes relacionadas ao AVC, a grande maioria em países desenvolvidos ${ }^{2}$. Tendo em vista o acelerado e intenso envelhecimento populacional brasileiro, estima-se que essa doença tenha cada vez mais relevância como um problema de saúde pública até que os investimentos em sua prevenção passem a ser prioridade do sistema de saúde brasileiro $^{3}$.

O AVC gera extensa variedade de déficits neurológicos funcionais - por exemplo, a perda do domínio voluntário dos movimentos musculares, problemas sensoriais, incontinência e dificuldades na comunicação e na fala ${ }^{4}$-, variantes conforme a localização da lesão, a dimensão da área de perfusão inadequada e a quantidade de fluxo sanguíneo colateral.

Nesse sentido, a incapacidade funcional é caracterizada pela dificuldade ou necessidade de ajuda para concretizar tarefas básicas de cuidados pessoais, denominadas atividades básicas de vida diária ${ }^{5}$.

Acredita-se que o tipo de AVC e o local anatômico da lesão implicam em déficits neurológicos de diferentes gravidades, considerando o nível de incapacidade funcional dos pacientes acometidos ${ }^{4,5}$.

Conhecer a capacidade funcional de pacientes pós-AVC e sua relação com o local de acometimento e o tipo de lesão pode ser importante ferramenta terapêutica no tratamento e na atenção de saúde desses pacientes, principalmente no que tange às políticas de reabilitação e tratamento clínico dessa população.

Esta pesquisa descreveu o perfil clínico e socioeconômico e a capacidade funcional de pacientes pós-AVC, considerando o tipo e a localização anatômica da lesão, atendidos em um ambulatório especializado.

\section{Método}

\section{Desenho do estudo e população}

Estudo transversal, quantitativo e descritivo, realizado no ambulatório de neurologia da Universidade Federal de Uberlândia (UFU-MG), com pacientes acometidos por AVC. Os pesquisadores em posse dos mapas de atendimento abordavam os pacientes agendados para consulta médica e avaliação clínica de rotina seguindo os critérios de inclusão: (1) idade superior a 18 anos; (2) diagnóstico médico de AVC confirmado em prontuário; (3) condiçôes cognitivas para responder aos instrumentos. Foram excluídos: (1) pacientes com diagnóstico médico inconclusivo; (2) internação menor de 30 dias. Após expor os objetivos da pesquisa, a participaçáo do paciente se formalizava perante a assinatura do termo de consentimento livre e esclarecido. O estudo foi aprovado pelo comitê de ética em pesquisa da instituição, sob no 277.825 .

\section{Desfecho do estudo}

Os desfechos deste estudo foram as escalas funcionais dos National Institutes of Health Stroke Scale (NIHSS) e do Índice de Barthel Modificado (IBM) dos pacientes acometidos pelo AVC.

\section{Entrevista clínica e socioeconômica}

Um questionário semiestruturado, adaptado pelos pesquisadores a partir de estudos publicados ${ }^{6,7,8,9}$, foi aplicado nos participantes, contendo as seguintes questốes socioeconômicas: sexo, idade, religiaao, nível de escolaridade, estado civil, condiçáo de trabalho e renda familiar. As questôes clínicas abordadas foram confirmadas em prontuário: tipo de $\mathrm{AVC}$, região anatômica acometida, antecedentes familiares de AVC, mobilidade, dificuldade de realizar tarefas e dificuldade de realizar atividades de lazer.

\section{Avaliação da severidade do AVC}

A NIHSS é uma escala que avalia sequelas causadas pelo AVC. Foi validada para uso no Brasi $^{6}$ por Cincura, Octavio e Pontes-Neto, em 2009, e é composta por onze itens, que incluem nível de consciência, movimentos oculares, campo visual, movimentos faciais, função motora, e ataxia de membros superiores e inferiores, assim como sensibilidade, linguagem, presença de disartria e de negligência espacial. Sua pontuação pode variar de 0 (sem evidência de déficit neurológico) a 42 (paciente em coma e sem resposta). De acordo com o escore obtido, pode-se predizer o tipo de acometimento neurológico, sendo que escore menor que 4 representa acometimento leve; escore de 4 a 20, acometimento moderado; escore maior que 20, acometimento grave ${ }^{8}$. 


\section{Avaliação da capacidade funcional}

O IBM é uma escala que mensura capacidade funcional, validada para uso no Brasil ${ }^{7}$ por Minosso, Amendola e Alvarenga em 2010. Sáo abordados dez aspectos básicos da atividade diária relacionados à mobilidade e aos cuidados pessoais: alimentação, higiene pessoal, controle dos esfíncteres vesical e intestinal, independência no banheiro, transferência da cadeira, marcha e capacidade para subir e descer escadas. A pontuação da escala varia de 0 a 100 , com intervalos de 5 pontos. A pontuação mínima corresponde à máxima dependência para todas as atividades de vida diária avaliadas, e a pontuação máxima equivale à independência total para os mesmos critérios avaliados ${ }^{6-9}$.

\section{Cálculo do tamanho amostral e análise estatística}

Considerando que no ambulatório de neurologia da UFU são atendidos aproximadamente cinco pacientes pós-AVC por mês (de acordo com o setor, dados disponibilizados pelo serviço), no período de três meses espera-se que sejam atendidos 60 pacientes. Além disso, considerando uma precisão amostral de 5\%, um nível de confiança de $95 \%$ e um $\mathrm{p}=0,5$, de acordo com metodologia sugerida por autores ${ }^{9}, n=\frac{Z^{2} p q N}{d^{2}(N-1)+Z^{2} p q}$, o resultado é de, no mínimo, $\mathrm{n}=14$ sujeitos de pesquisa.

Os dados foram importados eletronicamente para planilhas Microsoft Excel $^{\circ}$ e analisados pelo programa SPSS $^{\circ}$ (Statistical Package for the Social Sciences), versão 20.0. Foram realizadas análises descritivas.

\section{Resultados}

Foram selecionados e incluídos no estudo 14 pacientes. A amostra investigada foi caracterizada de acordo com os aspectos sociodemográficos (gênero, idade, religiāo, nível de escolaridade, estado civil, emprego, renda familiar, mobilidade, dificuldade de realizar tarefas e dificuldade de realizar atividades de lazer) e os aspectos clínicos (tipo do AVC, região anatômica cerebral acometida e histórico familiar de AVC).

Os resultados apontaram para uma amostra predominantemente masculina $(64,28 \%)$, com idades entre 18 e 55 anos. Em sua maioria, 78,57\% relataram dificuldade para subir escadas, abaixar, trocar lâmpadas, lavar roupas, lavar louças, cozinhar, ver televisão, ler, pegar objetos, movimentar, fazer contas, contar dinheiro, pegar ônibus, falar e dirigir. Esses e outros dados relevantes podem ser observados na Tabela 1 .

As características clínicas estão expostas na Tabela 2, em que se observa que o tipo de AVC mais prevalente foi o hemorrágico $(64,28 \%)$, seguido pelo isquêmico $(35,71 \%)$. Quanto às regiōes anatômicas, a frontal foi a mais comprometida $(42,85 \%)$.

Tabela 1: Características socioeconômicas dos participantes acometidos pelo AVC, atendidos no ambulatório de neurologia da Universidade Federal de Uberlândia, 2015.

\begin{tabular}{|c|c|c|}
\hline Variáveis & $\mathbf{N}$ & $\%$ \\
\hline \multicolumn{3}{|l|}{ Sexo } \\
\hline Masculino & 9 & 64,28 \\
\hline Feminino & 5 & 35,72 \\
\hline \multicolumn{3}{|l|}{ Idade } \\
\hline $18-40$ anos & 1 & 7,14 \\
\hline 41-60 anos & 8 & 57,14 \\
\hline $61-80$ anos & 5 & 35,72 \\
\hline \multicolumn{3}{|l|}{ Religiáo } \\
\hline Católica & 10 & 71,43 \\
\hline Evangélica & 4 & 28,57 \\
\hline \multicolumn{3}{|l|}{ Nível de escolaridade } \\
\hline Ensino fundamental - incompleto & 7 & 50,00 \\
\hline Ensino fundamental completo & 6 & 35,72 \\
\hline Ensino médio completo & 2 & 14,28 \\
\hline \multicolumn{3}{|l|}{ Estado civil } \\
\hline Casado & 7 & 50,00 \\
\hline Divorciado & 5 & 35,72 \\
\hline Viúvo & 2 & 14,28 \\
\hline \multicolumn{3}{|l|}{ Trabalho } \\
\hline Sem emprego fixo & 8 & 57,15 \\
\hline Possuem emprego fixo & 6 & 42,85 \\
\hline \multicolumn{3}{|l|}{ Renda familiar } \\
\hline Até 1 salário mínimo & 3 & 21,42 \\
\hline 1 a 3 salários mínimos & 6 & 42,85 \\
\hline 3 a 5 salários mínimos & 5 & 35,73 \\
\hline TOTAL & 14 & 100 \\
\hline
\end{tabular}

Fonte: Elaborado pelos autores.

Tabela 2: Características clínicas dos participantes acometidos pelo AVC, atendidos no ambulatório de neurologia da Universidade Federal de Uberlândia, 2015.

\begin{tabular}{|c|c|c|}
\hline Variáveis & $\mathbf{N}$ & $\%$ \\
\hline \multicolumn{3}{|l|}{ Tipo de AVC } \\
\hline Hemorrágico & 9 & 64,28 \\
\hline Isquêmico & 5 & 35,72 \\
\hline \multicolumn{3}{|l|}{ Regiáo anatômica acometida } \\
\hline Lobo frontal & 6 & 42,85 \\
\hline Lobo parietal & 4 & 28,59 \\
\hline Lobo occipital & 1 & 7,14 \\
\hline Lobo temporal & 1 & 7,14 \\
\hline Lobos temporal-parietal & 1 & 7,14 \\
\hline Cerebelar & 1 & 7,14 \\
\hline \multicolumn{3}{|c|}{ Antecedentes familiares de AVC } \\
\hline Sim & 8 & 57,15 \\
\hline Não & 6 & 42,85 \\
\hline \multicolumn{3}{|l|}{ Mobilidade } \\
\hline Independente & 9 & 64,28 \\
\hline Cadeirante & 5 & 35,72 \\
\hline \multicolumn{3}{|c|}{ Dificuldade de realizar tarefas } \\
\hline Sim & 11 & 78,57 \\
\hline Não & 3 & 21,43 \\
\hline \multicolumn{3}{|c|}{ Dificuldade de realizar atividades de lazer } \\
\hline Sim & 11 & 78,57 \\
\hline Não & 3 & 21,43 \\
\hline TOTAL & 14 & 100 \\
\hline
\end{tabular}

Fonte: Elaborado pelos autores. 
A NIHSS demonstra que boa parte dos pacientes avaliados $(42,85 \%)$ apresentavam um acometimento leve na severidade clínica e que a maioria $(57,14 \%)$ tinha sequelas moderadas. O IBM evidencia que a maioria dos pacientes $(64,28 \%)$ manifestou dependência leve, como mostra a Tabela 3.

Tabela 3: Características dos participantes quanto à severidade clínica (NIHSS) e à independência funcional (IBM), atendidos no ambulatório de neurologia da Universidade Federal de Uberlândia, 2015.

\begin{tabular}{lcc}
\multicolumn{1}{c}{ Variáveis } & N & $\%$ \\
NIHSS & 6 & 42,85 \\
Acometimento leve & 8 & 57,14 \\
Acometimento moderado & & \\
IBM & 9 & 64,28 \\
Dependência leve & 4 & 28,57 \\
Dependência moderada & 1 & 7,14 \\
Dependência severa & $\mathbf{1 4}$ & $\mathbf{1 0 0}$ \\
\hline TOTAL & & \\
\hline
\end{tabular}

Fonte: Elaborado pelos autores.
A relação entre a escala de severidade clínica (NIHSS) e a região anatômica da lesão demonstrou que, entre os pacientes que tiveram acometimento leve $(42,85 \%)$, a região anatômica com maior incidência foi a frontal $(21,42 \%)$. Entre os avaliados com acometimento moderado $(57,14 \%)$, a região mais acometida foi a parietal $(21,42 \%)$. Os pacientes com AVC do tipo hemorrágico demonstraram severidade clínica moderada (42,85\%). A Tabela 4 evidencia esses e outros dados referentes às relações estabelecidas.

Em referência à escala de capacidade funcional (IBM), nos pacientes com dependência leve a independência assistida $(64,28 \%)$ a região anatômica de maior ocorrência foi a frontal $(21,42 \%)$, enquanto os indivíduos avaliados com dependência moderada $(28,57 \%)$ foram acometidos em diversas regiōes anatômicas - parietal, temporal, bilateral frontal e cerebelar - nas mesmas proporçôes $(7,14 \%)$, como pode ser observado na Tabela 5.

Tabela 4: Relação entre escala de severidade clínica (NIHSS) com dados clínicos e sociodemográficos dos participantes atendidos no ambulatório de neurologia da Universidade Federal de Uberlândia, 2015.

\begin{tabular}{|c|c|c|c|}
\hline Variáveis & Acometimento leve (n, \%) & Acometimento moderado (n, \%) & Teste exato de Fisher (p) \\
\hline \multicolumn{4}{|l|}{ Regiáo anatômica } \\
\hline Frontal & $3(21,42)$ & $1(7,14)$ & - \\
\hline Occipital & $1(7,14)$ & - & - \\
\hline Parietal & $1(7,14)$ & $3(21,42)$ & - \\
\hline Temporal & - & $1(7,14)$ & - \\
\hline Temporal-parietal & - & $1(7,14)$ & - \\
\hline Frontal bilateral & $1(7,14)$ & $1(7,14)$ & - \\
\hline Cerebelo & - & $1(7,14)$ & - \\
\hline \multicolumn{4}{|l|}{ Tipo de AVC } \\
\hline Hemorrágico & $3(21,42)$ & $6(42,85)$ & 0,58 \\
\hline Isquêmico & $3(21,42)$ & $2(14,28)$ & \\
\hline \multicolumn{4}{|l|}{ AVC na família } \\
\hline Sim & $5(35,71)$ & $3(21,42)$ & 0,13 \\
\hline Não & $1(7,14)$ & $5(35,71)$ & \\
\hline \multicolumn{4}{|l|}{ Gênero } \\
\hline Masculino & $2(14,28)$ & $7(50,00)$ & 0,09 \\
\hline Feminino & $4(28,57)$ & $1(7,14)$ & \\
\hline \multicolumn{4}{|l|}{ Mobilidade } \\
\hline Cadeirante & $2(14,28)$ & $4(28,57)$ & 0,62 \\
\hline Independente & $4(28,57)$ & $4(28,57)$ & \\
\hline \multicolumn{4}{|c|}{ Dificuldade em realizar tarefas diárias } \\
\hline Sim & $5(35,71)$ & $6(42,85)$ & 1,00 \\
\hline Não & $1(7,14)$ & $2(14,28)$ & \\
\hline \multicolumn{4}{|c|}{ Dificuldade em realizar atividades de lazer } \\
\hline Sim & $4(28,57)$ & $7(50,00)$ & 0,53 \\
\hline Não & $2(14,28)$ & $1(7,14)$ & \\
\hline
\end{tabular}

Fonte: Elaborado pelos autores. 
Tabela 5: Relação entre o Índice de Barthel Modificado e as variáveis clínicas e socioeconômicas dos participantes atendidos no ambulatório de neurologia da Universidade Federal de Uberlândia, 2015.

\begin{tabular}{|c|c|c|c|c|}
\hline Variáveis & Dependência leve $\mathbf{n}(\%)$ & $\begin{array}{l}\text { Dependência moderada } \\
\qquad \mathbf{n}(\%)\end{array}$ & Dependência severa n(\%) & Teste exato de Fisher (p) \\
\hline \multicolumn{5}{|l|}{ Regiáo anatômica } \\
\hline Frontal & $4(28,57)$ & - & - & - \\
\hline Occipital & $1(7,14)$ & - & - & - \\
\hline Parietal & $2(14,28)$ & $1(7,14)$ & $1(7,14)$ & - \\
\hline Temporal & - & $1(7,14)$ & - & - \\
\hline Frontal bilateral & $2(7,14)$ & $1(7,14)$ & - & - \\
\hline Cerebelo & - & $1(7,14)$ & - & - \\
\hline \multicolumn{5}{|l|}{ Tipo de AVC } \\
\hline Hemorrágico & $6(42,85)$ & $2(14,28)$ & $1(7,14)$ & 0,53 \\
\hline Isquêmico & $3(21,42)$ & $2(14,28)$ & - & \\
\hline \multicolumn{5}{|l|}{$\begin{array}{l}\text { Antecedentes } \\
\text { familiares }\end{array}$} \\
\hline Sim & $6(42,85)$ & $1(7,14)$ & $1(7,14)$ & 0,53 \\
\hline Não & $3(21,42)$ & $3(21,42)$ & - & \\
\hline \multicolumn{5}{|l|}{ Gênero } \\
\hline Masculino & $5(35,71)$ & $4(28,57)$ & - & 1,00 \\
\hline Feminino & $4(28,57)$ & - & $1(7,14)$ & \\
\hline \multicolumn{5}{|l|}{ Mobilidade } \\
\hline Independente & $8(57,14)$ & $1(7,14)$ & - & 0,05 \\
\hline Cadeirante & $1(7,14)$ & $3(21,42)$ & $1(7,14)$ & \\
\hline \multicolumn{5}{|l|}{ Atividades de lazer } \\
\hline Sim & $7(50,00)$ & $3(21,42)$ & $1(7,14)$ & 0,85 \\
\hline Não & $2(14,28)$ & $1(7,14)$ & - & \\
\hline
\end{tabular}

Fonte: Elaborado pelos autores.

\section{Discussão}

Os dados deste estudo referentes ao acometimento do AVC em pacientes do sexo masculino corroboram um estudo previamente publicado, que pode ser explicado pelo fato de que homens possuem hábitos de vida menos saudáveis que mulheres, além de procurar os serviços de saúde com menor frequência9-10.

Em nosso estudo, pacientes avaliados com acometimento moderado pelo NIHSS e com dependência moderada pelo IBM eram, em sua maioria, do sexo masculino. Tal fato provavelmente pode ser explicado, pois, apesar de a incidência estar associada ao gênero, alguns estudos verificaram que ele não está aliado ao maior grau de incapacidade dos pacientes pós-AVC ${ }^{10}$.

Com relaçáo à faixa etária, neste estudo houve uma maior concentraçáo de pacientes do intervalo entre 41 a 60 anos $(57,14 \%)$, indo de encontro a pesquisas realizadas, segundo as quais em países em desenvolvimento o AVC ocorre cada vez mais cedo, relacionado à expectativa de vida e às condiçôes sociais desfavoráveis ${ }^{11,12}$.

Todos os pacientes responderam ter uma religiáo. A contribuiçáo desses achados positivos do envolvimento religioso pode ser buscada, do ponto de vista psicológico, na eficácia da religiâo em promover comportamentos saudáveis e em restringir comportamentos nocivos; na influência da religião nos estilos de vida pessoal; na integração e no apoio, favorecidos pelos atos religiosos sociais; na intensificação dos sentimentos de autoestima providos pela religiáo ${ }^{13,1,14,15}$.

Neste estudo observou-se que $64,28 \%$ dos pacientes eram independentes quanto à locomoção. Um estudo prévio evidenciou que a maioria dos pacientes acometidos por AVC $(60 \%$ a $75 \%)$ consegue caminhar, mas nem sempre são funcionalmente satisfatórios ${ }^{16}$.

Por meio da correlaçáo com as escalas de avaliaçáo funcional, neste estudo fica evidente que, além de a maior parte dos cadeirantes possuir um acometimento moderado (NIHSS), houve uma forte aproximaçáo dos critérios adotados para significância da relação dos déficits quanto à mobilidade e à presença de dependência moderada ou severa (IBM).

Quanto aos achados referentes à história familiar de AVC, cita-se que, apesar de tratar-se de um importante marcador para o risco de AVC, existe grande variabilidade de achados e poucos estudos epidemiológicos que avaliaram a hipótese de tal histórico como um fator de risco isolado ${ }^{17}$. 
Com relação ao tipo de AVC, constatou-se prevalência do hemorrágico $(64,28 \%)$ seguido do isquêmico $(35,71 \%)$, resultados divergentes de estudos observados na literatura, em que o tipo isquêmico é mais frequente em relação ao hemorrágico ${ }^{11,18,19}$.

Infere-se que a ocorrência desse resultado decorre da gravidade clínica dos pacientes que são atendidos no ambulatório em que foi realizada a pesquisa, por se tratar de uma instituiçáo universitária de referência em atendimento de pacientes graves.Em nosso estudo, $42,85 \%$ dos casos de AVC acometeram a região frontal do cérebro. Cabe ressaltar que os lobos frontais do córtex cerebral controlam as habilidades motoras aprendidas, como escrever e exercer atividades manuais corriqueiras, além de coordenar as expressóes faciais e os gestos expressivos ${ }^{20}$.

A lesão no lobo parietal $(28,57 \%)$ está relacionada a apraxia e incapacidade de realizar tarefas sequenciais e com incapacidade de reconhecer as partes do corpo ou o espaço ${ }^{20}$. Infere-se que esse tipo de lesão está totalmente associado com o prejuízo à realização de atividades basais e comuns ao ser humano que promovem o grau de independência funcional intrínseco a ele.

Os pacientes com lesão em lobo parietal apresentaram, mais comumente, acometimento e dependências mais severas, nas análises do resultado da avaliação funcional por meio das escalas de associação à região anatômica de acometimento.

\section{Conclusão}

O perfil clínico e sociodemográfico dos pacientes deste estudo concorda com a literatura da temática, dela divergindo apenas quanto à faixa etária de maior incidência, o que se deve ao fato de este ser um estudo de país em desenvolvimento.

O AVC do tipo hemorrágico esteve associado a piores resultados nas escalas de avaliação funcional, e as regióes anatômicas envolvidas com maior frequência foram os lobos cerebrais frontal e parietal, respectivamente, sendo que esse último, quando acometido, também foi o que apresentou maiores repercussóes para as atividades de vida diária dos pacientes, estando sempre relacionado à dependência moderada ou severa.

Infere-se a necessidade de estudos analíticos e experimentais que possam analisar de forma mais minuciosa os preditores e as variáveis referentes à capacidade funcional, ao tipo do AVC e à localizaçáo anatômica, uma vez que esses resultados podem auxiliar políticas públicas de saúde voltadas a essa população, assim como pode ser ferramenta estratégica para tratamento, acompanhamento e reabilitação desses pacientes.

\section{Referências}

1. Pompeu SMAA, Pompeu JE, Rosa M, Silva MR. Correlação entre função motora, equilíbrio e força respiratória pós acidente vascular cerebral. Rev Neurocienc. 2011;19(4):614-20.

2. Gomes-Neto M. Aplicação da escala de qualidade de vida especifica para AVE (EQVE-AVE) em hemiplégicos agudos: propriedades psicométricas e sua correlação com a classificação internacional de funcionalidade, incapacidade e saúde [dissertação de mestrado]. Belo Horizonte: Universidade Federal de Minas Gerais; 2007.

3. André C. Manual de AVC. 2. ed. Rio de Janeiro: Revinter; 2006.

4. Parahybama VR. Diferenciais sociodemográficos no declínio funcional em mobilidade física entre idosos no Brasil. Ciênc Saúde Colet. 2008;13(4):1257-64.

5. Cincura C, Pontes-Neto OM, Neville IS, Mendes HF, Menezes DF, Mariano DC, et al. Validation of the National Institutes of Health Stroke Scale, modified Rankin Scale and Barthel Index in Brazil: the role of cultural adaptation and structured interviewing. Cerebrovasc Dis. 2009;27(2):119-22.

6. Minosso JSM, Amendola F, Alvarenga MRM, Oliveira MAC. Validação, no Brasil, do Índice de Barthel em idosos atendidos em ambulatórios. Acta Paul Enferm. 2010;23(2):218-23.

7. Jauch EC, Saber JL, Adams HP, Bruno A, Connors JJ, Demaerschalk BM, et al. Guidelines for the early management of patients with acute ischemic stroke: a guideline for healthcare professionals from the American Heart Association/American Stroke Association. Stroke. 2013 Mar;44(3):870-947.

8. Walter J, Grave MTQ, Périco E. Avaliação das habilidades psicomotoras e da motricidade global em paciente portadora da doença de Huntington. ConScientiae Saúde. 2009;8(4):655-63.

9. Fonseca JS, Martins GA. Curso de estatística. 6. ed. São Paulo: Atlas; 2006.

10. Sidney S, Rosamond WD, Howard VJ, Luepker RV. The "heart disease and stroke statistics-2013 update" and the need for a national cardiovascular surveillance system. Circulation. 2013 Jan;127(1):21-3.

11. Kelly-Hayes M, Beiser A, Kase CS, Scaramucci A, D'Agostino RB, Wolf PA. The influence of gender and age on disability following ischemic stroke: the Framingham study. J Stroke Cerebrovasc Dis. 2003 May-Jun;12(3):119-26.

12. Minelli C, Fen LF, Minelli DP. Stroke incidence, prognosis, 30-day, and 1-year case fatality rates in Matão, Brazil: a population-based prospective study. Stroke. 2007 Nov;38(11):2906-11.

13. Confort AB, Suely KN, Marie, LG, Cohen, MS. Estimulação magnética transcraniana. Arq Neuro-Psiquiatr. 2003 mar;61(1):146-52.

14. Cohen S, Herbert, TB. Health psychology: psychological factors and physical disease from the perspective of 
human psychoneuroimmunology. Annu Rev Psychol. 1996;47(1):113-42.

15. Ellison CG. Introduction to symposium: religion, health and well-being. Journal for the Scientific Study of Religion. 1998;37(4):692-4.

16. Kiecolt-Glaser JK, McGuire L, RoblesTF, Glaser R. Emotions, morbidity, and mortality: new perspectives from psychoneuroimmunology. Annu Rev Psychol. 2002;53:83-107.
17. Ottoboni C, Fontes SV, Fukujima MM. Estudo comparativo entre a marcha normal e a de pacientes hemiparéticos por acidente vascular encefálico: aspectos biomecânicos. Rev Neurociências. 2002;10(1):10-6.

18. Kubota JT, Banaji MR, Phelps EA. The neuroscience of race. Nature Neurosci. 2012;15:940-8.

\section{Como citar este artigo:}

Souza NR, Caldeira LPN, De Almeida Neto OP, Pereira MER. Capacidade funcional, etiologia e local anatômico da lesão no acidente vascular cerebral. Rev. Aten. Saúde. 2016;14(49):59-65. 